\title{
Weak interaction in the African Easterly Jet
}

\author{
A. Lenouo ${ }^{1}$, F. Nkankam Kamga ${ }^{1}$, and E. Yepdjuo ${ }^{2}$ \\ ${ }^{1}$ Atmospheric Sciences Lab. Dept. of Physics, Univ. of Yaounde I, Cameroon P.O. Box 812 Yaoundé, Cameroon, Africa \\ ${ }^{2}$ EAMAC, P.O. Box 746, Niamey, Niger, Africa
}

Received: 27 May 2004 - Revised: 4 April 2005 - Accepted: 8 April 2005 - Published: 28 July 2005

\begin{abstract}
Low-frequency, African Easterlies Waves (AEW) are examined as disturbances embedded in the midtropospheric easterly jet of the African low troposphere. The solution to the nonlinear vorticity equation relevant to the description of waves is sought in the form of triplet waves. The latest suggest a unified method to determine their kinetics characteristic and to explain the mechanism of energy exchange between their different modes. The period of energy interaction between different modes of the global wave is equal to 3.5 days when the wave packet is moving with a group velocity dependent on the mean basic flow. The effects of nonlinearity are also identified, and it is noted that the horizontal shears of mean flow, as well as the temporal variation of the amplitude wave functions, are the controlling factors.
\end{abstract}

Keywords. Meteorology and atmospheric dynamics (Synoptic-scale meteorology; Tropical meteorology; Waves and tides)

\section{Introduction}

GATE and other field experiments have produced a wealth of results leading to a fairly clear image of the mean vertical structure of the tropical atmosphere over Africa. In the 1970's, two jet streams were identified by Burpee (1972) from the analysis of the vertical structure of the mean zonal wind. The first one, the African Easterly Jet (AEJ), is located around $3 \mathrm{~km}$ height (600 to $700 \mathrm{hPa}$ ) in the lower troposphere. The second one, the Tropical Easterly Jet (TEJ), is found at an altitude of $18 \mathrm{~km}$. Kwon (1989) has presented the vertical profile of the zonal component of the wind, derived from a semi-empirical expression calibrated using West African meteorological data (Fig. 1a). Although the vertical positions of the jets are in agreement with early analyses, their latitudinal positions are not apparent. Similar profiles, based on experimental data, were presented by Burpee (1972) (Fig. 1b). This profile clearly shows that the

Correspondence to: A. Lenouo

(lenouo@yahoo.fr) axis of AEJ is around $15^{\circ} \mathrm{N}$ and $700 \mathrm{hPa}$ position (meridian and vertical) of order jet.

It is well established that the tropical atmosphere is a region of large-scale perturbations that develop and propagate eastward. Much attention has been focused on these perturbations because of their role in maintaining the general circulation of the atmosphere and in modulating precipitation. Theoretical and experimental evidence have established their characteristics and the dominant role of barotropic instability at their genesis. In the African low troposphere, some of these disturbances are associated with the mid-tropospheric jet known as the easterly wave (Burpee, 1972). The characteristics of easterly waves, located around $600-700 \mathrm{hPa}$ in the African low troposphere, have widely been studied in the past (Rennick, 1976; Mass, 1979; Kwon, 1989; Thorncroft et al., 1994a,b).

By considering a linear primitive equations (PE) model and a quasi-geostrophic (QG) model, these authors have determined their kinematic characteristics and the dominant energy source in the region. Using a PE model, Rennick (1976) showed that near the jet core, the wavelength is about $3000 \mathrm{~km}$, the phase velocity $16 \mathrm{~m} \cdot \mathrm{s}^{-1}$, and the period has the value 2.2 days for a maximum zonal wind on the order of $16 \mathrm{~m} \cdot \mathrm{s}^{-1}$. Similarly, Mass (1979) obtained values of $2500 \mathrm{~km}, 7.5 \mathrm{~m} . \mathrm{s}^{-1}, 3.8$ days and $13 \mathrm{~m} . \mathrm{s}^{-1}$, respectively. On the QG model side, the numerical analysis made by Kwon (1989) showed that in the barotropic instability zone, where the zonal wind at $625 \mathrm{hPa}$ is of the order of $14 \mathrm{~m} . \mathrm{s}^{-1}$, the respective characteristics for easterly waves are $3000 \mathrm{~km}, 10 \mathrm{~m} \cdot \mathrm{s}^{-1}$ and 3.5 days. The linear and nonlinear instability problem of the African Easterly Jet (AEJ) has been investigated by Thorncroft et al. (1994, 1995), using the PE model on a sphere. For a basic flow with maximum wind of about $15 \mathrm{~m} . \mathrm{s}^{-1}$ at $600 \mathrm{hPa}$, they showed that for a wavelength of about $3500 \mathrm{~km}$, a phase velocity of $6.8 \mathrm{deg} / \mathrm{day}$ is obtained, implying a period of about 4.8 days.

Wegiel and Herbster (2000) have proposed one possible mechanism responsible for the origin of the African Easterly Wave (AEW). A model simulation was performed documenting the orogenesis of meso-scale systems in Ethiopia. They demonstrated that the dynamical structure and maintenance 

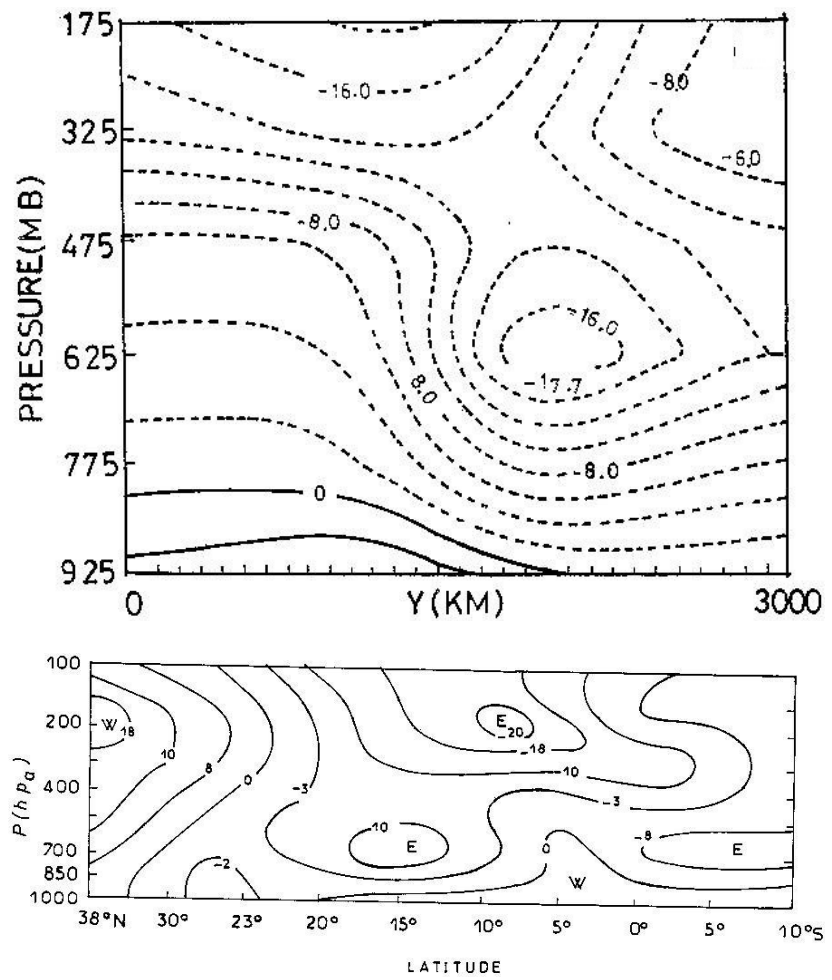

Fig. 1. Mean zonal wind profiles over West Africa during the summer period: adapted from (a) Kwon (1989) and (b) Burpee (1972). Units are $\mathrm{m} . \mathrm{s}^{-1}$. Figure $1(\mathrm{~b})$ indicates that the AEJ is centred over $15^{\circ} \mathrm{N}$ at around $700 \mathrm{hPa}$.

of these synoptic-scale easterly perturbations can be simulated with a great deal of accuracy. They also showed that the Ethiopian Highlands act as the source region for AEWs.

However, these authors did not fully take into account group velocity, which, in our opinion, is an essential parameter in the understanding of AEW. Group velocity can be non zero, even when the phase velocity is zero, e.g. in the case of stationary Rossby waves (Hoskin and Ambrizzi, 1993). Furthermore, the study of the dynamics of perturbations associated with the easterly jet in the lower troposphere is, in many aspects, similar to that of Rossby waves. Indeed, one uses the nonlinear vorticity equation in a horizontal plane. However, most published work does not account for some of the fundamental properties related to the nonlinearity, and in particular, energy transfer between waves.

Numerous experimental observations have pointed to the possibility of interactions between atmospheric waves in the tropical zone (Dobryshman, 1982), as well as in the extratropical regions (McIntyre and Palmer, 1983). Theoretical treatment of this type of interacting waves was done for small-scale waves in the equatorial zone by Dobryshman (1982) and for planetary waves in the stratosphere by McIntyre and Palmer (1983). They considered a weakly interacting wave triplet at resonance. Then, using the triangular relation between the wave numbers of the triplet, they suggested an analytical amplitude function appropriate for the description of the waves under consideration. However, the explanation of the energy exchange mechanism between waves was partial and unsatisfactory.

In the present study, the hypothesis of weak interaction at resonance between the waves of the triplet is maintained. We use a method developed by Longuet-Higgins and Hill (1970), to solve analytically the solution to the nonlinear vorticity equation, subject to the conservation of the total energy of the system. We are able to show how these three waves account, under weak interaction, for the energy transfer between waves, leading to energy transport in the phase space of wave numbers (Kadomtsev, 1979). We also indicate a technique to determine the characteristics of group velocity, i.e. the velocity of energy transfer by the basic waves around the jet. This work is done by considering energy transfer between waves in the horizontal plane, especially at the $700 \mathrm{hPa}$ level, where AEJ is located. The barotropic theory was also used by Ferreira and Schubert (1997) to study the climatologic aspect of West Africa region through the Intertropical Convergence Zone (ITCZ).

Section 2 is a presentation of the model used in this study. Analytic expressions of the amplitude functions of the waves in interaction are obtained in Sect. 3. Section 4 is devoted to results and discussions.

\section{The model}

\subsection{Model formulation}

Since the vertical extension of AEJ is not important, AEW can be regarded, in the first approximation, as a horizontal motion at jet level. To analyse the resultant perturbations, we consider a two-dimensional model for an incompressible and inviscid fluid in the $\beta$-plane; horizontal coordinates (x,y) are chosen such that the $\mathrm{x}$ axis is oriented eastward and $\mathrm{y}$ northward. The model is governed by the following equations:

$$
\begin{aligned}
& \left(\frac{\partial}{\partial t}+u \frac{\partial}{\partial x}+v \frac{\partial}{\partial y}\right) u-f v=-\frac{\partial \phi}{\partial x} \\
& \left(\frac{\partial}{\partial t}+u \frac{\partial}{\partial x}+v \frac{\partial}{\partial y}\right) v+f u=-\frac{\partial \phi}{\partial y} \\
& \frac{\partial u}{\partial x}+\frac{\partial v}{\partial y}=0,
\end{aligned}
$$

where $u$ and $v$ are the zonal and meridional wind components, $f$ the Coriolis parameter, $\phi$ the geopotential and $t$ the time. The current method of solving system (1) through the decomposition of the dependant variables in terms of mean basic states and of small perturbations is employed. We therefore write $\mathrm{u}=\mathrm{U}(\mathrm{y})+\mathrm{u}^{\prime}$ and $\mathrm{v}=\mathrm{v}$, where the prime terms refer to perturbations and the capital $\mathrm{U}$ refers to the basic flow.

The following expression for the horizontal sheared zonal component of the wind, proposed by Rennick (1976), is used in our analysis:

$U(y)=-U_{0} \sin ^{2}\left(\pi \mathrm{y} / 2 \mathrm{y}_{0}\right)$, 
where $\mathrm{U}_{0}=20 \mathrm{~m} . \mathrm{s}^{-1}$; $\mathrm{y}_{0}$ corresponds to the latitude of the jet axis, the origin of latitude $(y=0)$ is located at the equator. $\mathrm{U}(\mathrm{y})$ is symmetric about the axis of the jet $\left(\mathrm{y}=\mathrm{y}_{0}\right)$, having a maximum on it and decreasing away from this axis, to zero on the equator $(\mathrm{y}=0)$ and at a latitude of $30^{\circ} \mathrm{N}\left(\mathrm{y}=2 \mathrm{y}_{0}\right)$ (Fig. 5).

Since the flow is quite non divergent, we introduce a stream function, $\psi$, so that

$u^{\prime}=-\frac{\partial \psi}{\partial y}$ and $\mathrm{v}^{\prime}=\frac{\partial \psi}{\partial \mathrm{x}}$.

Substituting in the expression for the curl of the velocity vector, whose components (u,v) appear in Eqs. (1.1) and (1.2), we obtain an equation for the vorticity related to the perturbation, in the form

$$
\left(\frac{\partial}{\partial t}+U \frac{\partial}{\partial x}\right) \nabla^{2} \psi+J\left(\psi, \nabla^{2} \psi\right)+\left(\beta-\frac{d^{2} U}{d y^{2}}\right) \frac{\partial \psi}{\partial x}=0
$$

where

$$
J(A, B)=\frac{\partial A}{\partial x} \frac{\partial B}{\partial y}-\frac{\partial A}{\partial y} \frac{\partial B}{\partial x}
$$

is the Jacobian operator, $\nabla^{2}$ the Laplacian, $\left(\nabla^{2}=\partial^{2} / \partial \mathrm{x}^{2}+\partial^{2} / \partial \mathrm{y}^{2}\right)$, and $\beta$ the meridional gradient of the Coriolis parameter, $(\beta=\partial \mathrm{f} / \partial \mathrm{y})$.

\subsection{Energy conservation equation}

Solutions to Eq. (3) must satisfy the principle of conservation of total energy, which, on the horizontal plane, reduces to the conservation of kinetic energy. Let $\varepsilon$ be the total energy per unit mass of fluid. Multiplying Eq. (3) by $\psi$, one obtains, after some algebra, the following local equation for energy:

$\varepsilon=\frac{1}{2} \frac{\partial}{\partial t}(\nabla \psi \cdot)^{2}$

\section{Solution to the model equation}

A general solution to Eq. (3) is sought in the form:

$\psi(x, y, t)=\sum_{k=1}^{3} \psi_{k}(t) \exp -i\left(m_{k} x+n_{k} y-\sigma_{k} t\right)$,

where $m_{k}$ and $n_{k}$ are zonal and meridional wave numbers, respectively; $\sigma_{k}$ is the growth rate and $\psi_{k}(\mathrm{t})$, the amplitude time function of the $\mathrm{k}^{\text {th }}$ component of the wave. Substituting Eq. (5) into Eq. (3), leads to the following equation:

$$
\begin{aligned}
& -\left(m_{k}^{2}+n_{k}^{2}\right) \frac{\partial \psi_{k}}{\partial t}-i\left(m_{k}^{2}+n_{k}^{2}\right)\left(\sigma_{k}-m_{k} U\right) \psi_{k} \\
& +m_{k} \psi_{k} \sum_{j=1}^{3}\left[n_{j}\left(m_{j}^{2}+n_{j}^{2}\right) \psi_{j}\right] \\
& -\left[m_{k}\left(m_{k}^{2}+n_{k}^{2}\right) \psi_{k}\right] \sum_{j=1}^{3} n_{j} \psi_{j} \\
& -i m_{k}\left(\beta-\frac{d^{2} U}{d y^{2}}\right) \psi_{k}=0
\end{aligned}
$$

with $\mathrm{k} \equiv 1,2,3$.

By identifying terms, the following relations are derived: (i) conditions for resonance,

$$
\begin{aligned}
& m_{1}+m_{2}+m_{3}=0 \\
& n_{1}+n_{2}+n_{3}=0 \\
& \sigma_{1}+\sigma_{2}+\sigma_{3}=0
\end{aligned}
$$

(ii) the dispersion equation,

$$
\sigma_{k}=m_{k} U-\left(\beta-\frac{d^{2} U}{d y^{2}}\right) \frac{m_{k}}{m_{k}^{2}+n_{k}^{2}}
$$

(iii) and the nonlinear equation for amplitude function, $\psi_{k}(\mathrm{t})$,

$$
\begin{gathered}
\left(m_{k}^{2}+n_{k}^{2}\right) \frac{\partial \psi_{k}}{\partial t}=m_{k} \psi_{k} \sum_{j=1}^{3}\left[n_{j}\left(m_{j}^{2}+n_{j}^{2}\right) \psi_{j}\right] \\
-\left[m_{k}\left(m_{k}^{2}+n_{k}^{2}\right) \psi_{k}\right] \sum_{j=1}^{3} n_{j} \psi_{j}
\end{gathered}
$$

$\mathrm{k} \equiv 1,2,3$.

Setting,

$\rho_{k}^{2}=m_{k}^{2}+n_{k}^{2} a$ and $\eta_{k}=m_{k+1} n_{k-1}-m_{k-1} n_{k+1}$,

with the convention $(\mathrm{m}, \mathrm{n})_{0}=(\mathrm{m}, \mathrm{n})_{3}$ and $(\mathrm{m}, \mathrm{n})_{4}=(\mathrm{m}, \mathrm{n})_{1}$, and substituting in Eq. (8), leads to the following uncoupled system of equations (see Dobryshman, 1982; McIntyre and Palmer, 1983; and Pedlosky, 1998):

$$
\begin{aligned}
& \rho_{1}^{2} \frac{d \psi_{1}}{d t}=\eta_{1}\left(\rho_{3}^{2}-\rho_{2}^{2}\right) \psi_{2} \psi_{3} \\
& \rho_{2}^{2} \frac{d \psi_{2}}{d t}=\eta_{2}\left(\rho_{1}^{2}-\rho_{3}^{2}\right) \psi_{1} \psi_{3} \\
& \rho_{3}^{2} \frac{d \psi_{3}}{d t}=\eta_{3}\left(\rho_{2}^{2}-\rho_{1}^{2}\right) \psi_{2} \psi_{1} .
\end{aligned}
$$

On the other hand, when $\psi$ given by Eq. (5) is substituted into the energy Eq. (4), one obtains

$\varepsilon=\rho_{k}^{2}\left[\sigma_{k} \psi_{k}^{2}+\psi_{k} \frac{\partial \psi_{k}}{\partial t}\right]$,

which, upon using the set of Eq. (9), becomes:

$$
\begin{aligned}
& \varepsilon=\rho_{1}^{2} \sigma_{1} \psi_{1}^{2}+\rho_{2}^{2} \sigma_{2} \psi_{2}^{2}+\rho_{3}^{2} \sigma_{3} \psi_{3}^{2}+\eta_{1}\left(\rho_{3}^{2}-\rho_{2}^{2}\right) \psi_{1} \psi_{2} \psi_{3} \\
& +\eta_{2}\left(\rho_{1}^{2}-\rho_{3}^{2}\right) \psi_{1} \psi_{2} \psi_{3}+\eta_{3}\left(\rho_{2}^{2}-\rho_{1}^{2}\right) \psi_{1} \psi_{2} \psi_{3} .
\end{aligned}
$$

It is readily verified, in view of relation Eq. (6), that

$\eta_{2}-\eta_{1}=0 ; \eta_{2}-\eta_{3}=0$ and $\eta_{3}-\eta_{1}=0$.

Thus,

$\eta_{1}=\eta_{2}=\eta_{3}=\eta$.

The expression for total energy is now reduced to

$\varepsilon=\rho_{1}^{2} \sigma_{1} \psi_{1}^{2}+\rho_{2}^{2} \sigma_{2} \psi_{2}^{2}+\rho_{3}^{2} \sigma_{3} \psi_{3}^{2}$.

Furthermore, the system of Eq. (9) can be rewritten as:

$\frac{d \overline{\psi_{1}}}{d t}=\overline{\psi_{2} \psi_{3}} ; \frac{d \overline{\psi_{2}}}{d t}=-\overline{\psi_{1} \psi_{3}}$ and $\frac{\mathrm{d} \overline{\psi_{3}}}{\mathrm{dt}}=\overline{\psi_{2} \psi_{1}}$; 
with $\bar{\psi}_{k}(\mathrm{t})=\psi_{k}(\mathrm{t}) p_{k}^{-1}, \mathrm{k} \equiv 1,2,3$; and $\mathrm{p}_{k}$ is given by

$$
\begin{aligned}
& p_{1}=\frac{\rho_{2} \rho_{3}}{\eta \sqrt{\left(\rho_{2}^{2}-\rho_{1}^{2}\right)\left(\rho_{3}^{2}-\rho_{1}^{2}\right)}}, \\
& p_{2}=\frac{\rho_{1} \rho_{3}}{\eta \sqrt{\left(\rho_{3}^{2}-\rho_{2}^{2}\right)\left(\rho_{2}^{2}-\rho_{1}^{2}\right)}}
\end{aligned}
$$

and

$$
p_{3}=\frac{\rho_{2} \rho_{1}}{\eta \sqrt{\left(\rho_{3}^{2}-\rho_{1}^{2}\right)\left(\rho_{3}^{2}-\rho_{2}^{2}\right)}}
$$

after reordering the indices so that $\rho_{1}<\rho_{2}<\rho_{3}$.

The first two equations of Eq. (11) are combined to give

$\overline{\psi_{1}} \frac{d \overline{\psi_{1}}}{d t}+\overline{\psi_{2}} \frac{d \overline{\psi_{2}}}{d t}=0$.

By direct integration, one obtains

$\bar{\psi}_{1}^{2}+\bar{\psi}_{2}^{2}=N_{1}^{2}=$ constant.

A similar combination of the second and third equations of Eq. (11) gives

$\bar{\psi}_{3}^{2}+\bar{\psi}_{2}^{2}=N_{2}^{2}=$ constant.

Using the last two relations, $\bar{\psi}_{1}$ and $\bar{\psi}_{3}$ are eliminated from the second equation of Eq. (11), expression of $\bar{\psi}_{2}$ is obtained from the following equation:

$\frac{d \overline{\psi_{2}}}{d t}=\sqrt{\left(N_{1}^{2}-\bar{\psi}_{2}^{2}\right)\left(N_{2}^{2}-\bar{\psi}_{2}^{2}\right)}$.

The solution for $\bar{\psi}_{2}$, subject to the initial condition $\bar{\psi}_{2}\left(\mathrm{t}_{0}=0\right)=0$, is an elliptic Jacobian function of the form (M. Abromowitz and Segun, 1964)

$\bar{\psi}_{2}(t)=N_{2} \operatorname{sn}(t / \alpha)$

with $\alpha=\operatorname{Arcsin}\left(\mathrm{N}_{2} / \mathrm{N}_{1}\right)$.

$\bar{\psi}_{1}(\mathrm{t})$ and $\bar{\psi}_{3}(\mathrm{t})$ are obtained from Eqs. (12) and (13), respectively, as:

$\bar{\psi}_{1}(t)=N_{1} d n(t / \alpha)$,

$\bar{\psi}_{3}(t)=N_{2} c n(t / \alpha)$.

These amplitude functions are periodic and their period depends on the parameter $\alpha$. It is calculated from the following relation

$$
K=\int_{0}^{\pi / 2} \frac{d \tau}{\sqrt{1-\left(N_{2} / N_{1}\right)^{2} \sin ^{2} \tau}},
$$

where $K$ is one quarter of the period of the Jacobian elliptic function and depends only on $N_{2} / N_{1} . K$ is plotted in Fig. 2 . Its value increases slowly for values of $N_{2} / N_{1}$ less than 0.9 and grows rapidly to $\infty$ as $N_{2} / N_{1}$ goes to unity. It follows that the interaction time between triplets is of the order of several days.

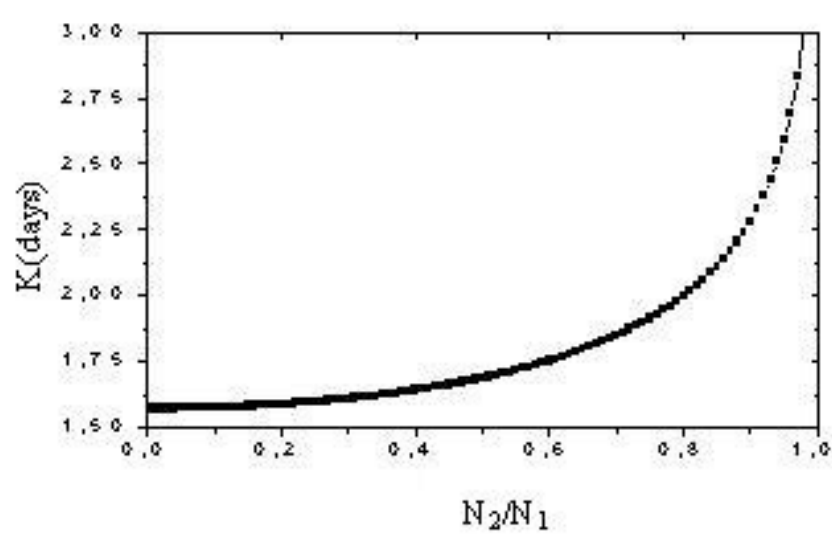

Fig. 2. Dependence of energy interaction time, $\mathrm{K}$, on the ratio $\mathrm{N}_{2} / \mathrm{N}_{1}$.

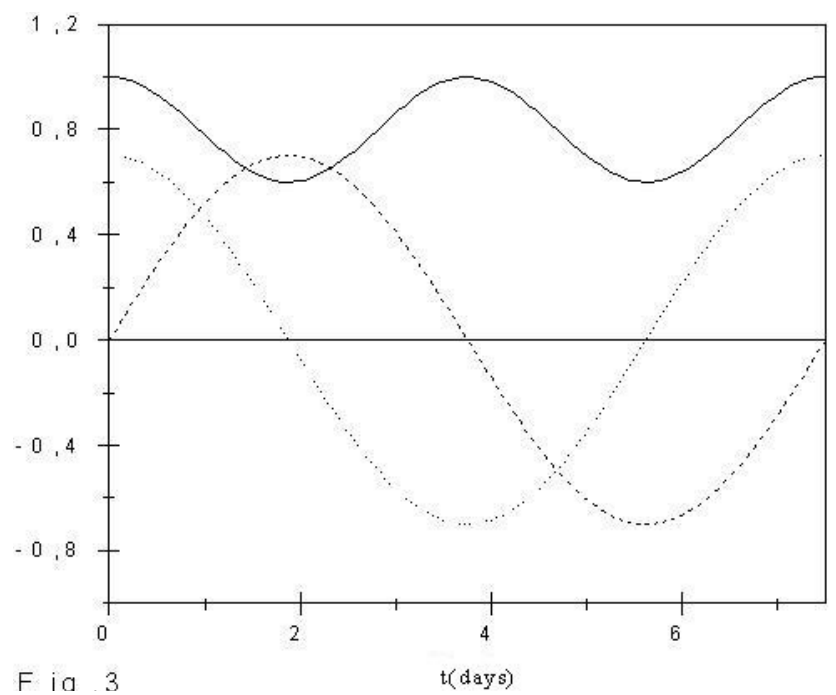

Fig. 3. Time evolution of the amplitude $\bar{\psi}_{k}(\mathrm{t})$ of the $\mathrm{k}^{t h}$ wave for $\mathrm{N}_{2} / \mathrm{N}_{1}=0.7$ for a period of 7.5 days. The amplitude $\bar{\psi}_{1}$ (solid line) has the same period as the AEW, whereas the amplitudes $\bar{\psi}_{2}$ (dash) and $\bar{\psi}_{3}(\mathrm{dot})$ have twice this period.

\section{Discussions}

\subsection{Interaction between waves}

It is important to note that all parameters in Eq. (6) are non zero. Otherwise, the wave triplet would be reduced to a doublet. We would then have $\rho^{2}=m_{1}^{2}+n_{1}^{2}=m_{2}^{2}+n_{2}^{2}$ and $\psi_{k}=$ constant for $\mathrm{k} \equiv 1,2$, and no interactions between waves, since the interactions are controlled by the interdependence of the amplitude function of the waves. Moreover, these would be purely linear waves.

An additional condition for wave interaction is that no two parameters $\psi_{k}(\mathrm{k}=1,2,3)$ be equal, e.g. if $\rho_{1}=\rho_{2}$, then from Eq. (9), $\psi_{3}$ (constant) would not interact with $\psi_{1}$ or $\psi_{2}$.

In the wave triplet case, the amplitude function $\bar{\psi}_{k}(\mathrm{t})$, $(\mathrm{k}=1,2,3)$, is plotted in Fig. 3 for a time span of 7.5 days, with $N_{2} / N_{1}$ set at 0.7 . This period should not be mistaken 
Table 1. Possible combinations of the signs of the triplet $\left(\sigma_{1}, \sigma_{2}\right.$, $\sigma_{3}$ ) and their effect on energy conservation.

\begin{tabular}{cccc}
\hline$\sigma_{1}$ & $\sigma_{2}$ & $\sigma_{3}$ & $\varepsilon$ \\
\hline+ & + & + & $\mathrm{N}$ \\
+ & + & - & $\mathrm{N}$ \\
+ & - & + & $\mathrm{Y}$ \\
+ & - & - & $\mathrm{N}$ \\
- & + & + & $\mathrm{N}$ \\
- & + & - & $\mathrm{Y}$ \\
- & - & + & $\mathrm{N}$ \\
- & - & - & $\mathrm{N}$ \\
\hline
\end{tabular}

for that of the global waves $\psi$, which has mean values of 2 to 3.4 days, in the case of AEW. Instead, it is the period of the amplitude functions representing the time necessary for complete energy exchange between waves. These results are similar in many aspects to those obtained by Dobryshman (1982), Pedlosky (1998) and Vanneste and Vial (1995) in their studies of small-scale waves to non constant background flows.

Let us now examine more conveniently the energy interactions. From Eq. (10), total energy is given by

$\varepsilon=\varepsilon_{1}+\varepsilon_{2}+\varepsilon_{3}$,

with $\varepsilon_{k}$ being the $\mathrm{k}^{\text {th }}$ wave energy normalised by $\rho_{k}^{2} p_{k}^{2}$ (Kadomtsev, 1979), so that

$\varepsilon_{k}=\sigma_{k} \bar{\psi}_{k}^{2}, k=1,2,3$.

Table 1 shows the proper choice of the sign of $\sigma_{k}$ for energy to be conserved. A letter $\mathrm{Y}$ in the last column indicates the two acceptable combinations. $\sigma_{1}$ and $\sigma_{3}$ must have the same sign and $\sigma_{2}$ the opposite sign. The letter $\mathrm{N}$ indicates the case where the energy is not conserved.

Taking into account these conditions and using Eq. (6), the total energy is rewritten as:

$\varepsilon=\sigma_{1} N_{1}^{2}+\sigma_{3} N_{2}^{2}$.

We note that $\varepsilon$ depends on $\sigma_{1}, \sigma_{3}, N_{1}$ and $N_{2}$ and not on time t.

The duration of the interaction between waves is given by half of the period of the elliptic function $(2 K)$, since $\varepsilon_{k}$ is proportional to $\bar{\psi}_{k}^{2}$.

The energy $\varepsilon_{k}$ of each wave as a function of time for $\mathrm{N}_{2} / \mathrm{N}_{1}=0.7$ is shown in Fig. $4 \mathrm{a}$ for $\sigma_{1}=1, \sigma_{2}=-1.5$ and $\sigma_{3}=0.5$. These functions have a period of approximately $2 \mathrm{~K}=3.7$ days. It is noted that $\varepsilon_{1}$ and $\varepsilon_{3}$ are in phase and are both out of phase with $\varepsilon_{2}, \varepsilon_{1}$ and $\varepsilon_{3}$ lose energy in the first half of the cycle, while $\varepsilon_{2}$ is gaining, and the reverse is true in the second half. Thus, in a three wave process, energy is constantly being exchanged between waves in such a way that the total energy is conserved. It is important to note that this period is equal to those of $\mathrm{AEW}$.
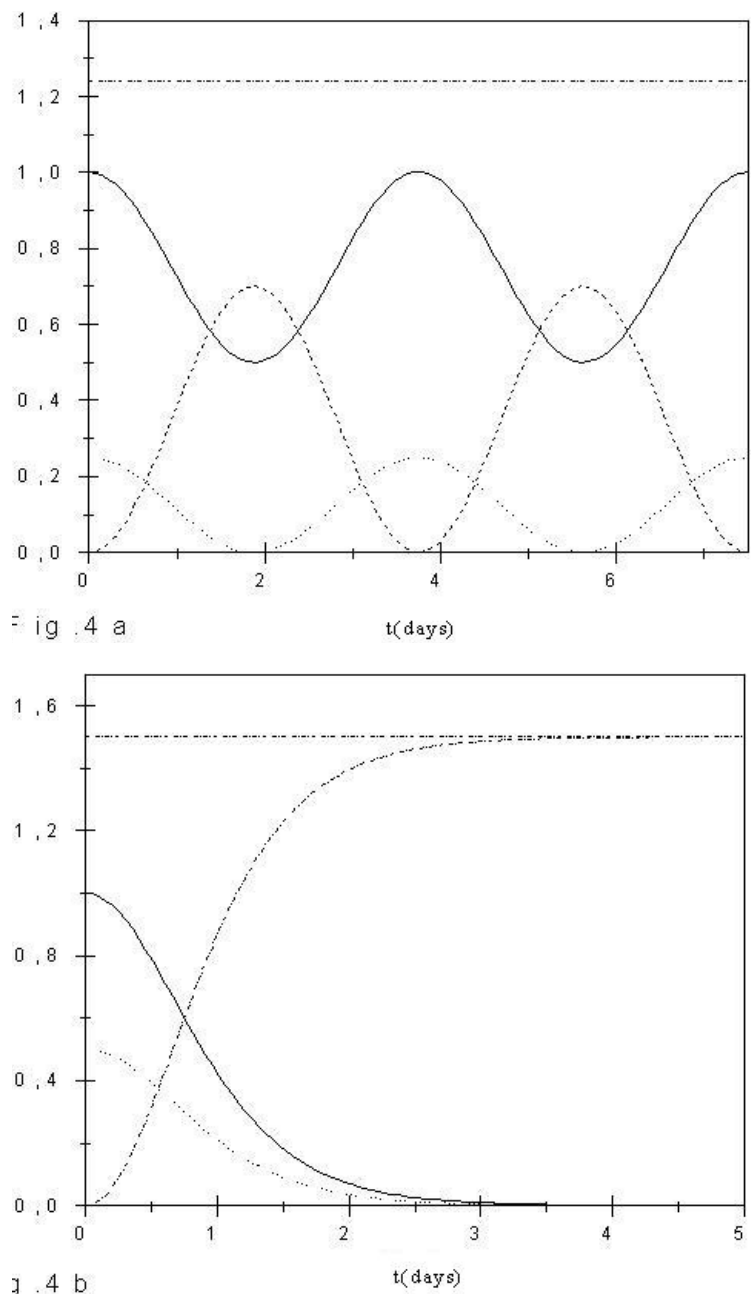

Fig. 4. Energy of the waves as a function of time for (a) $\mathrm{N}_{2} / \mathrm{N}_{1}=0.7$ and (b) $N_{1}=N_{2}=1 . \varepsilon_{1}$ (in solid), $\varepsilon_{2}$ (in dash), $\varepsilon_{3}$ (in dot) and $\varepsilon$ (in dash dot). Total energy, $\varepsilon$, is constant over the time.

This energy exchange process is more interesting in the limiting case when $\mathrm{N}_{1}=\mathrm{N}_{2}=1$, then the amplitude functions are

$\bar{\psi}_{1}(t)=\operatorname{Sech}(\mathrm{t}) ; \bar{\psi}_{2}(\mathrm{t})=\tanh (\mathrm{t})$ and $\bar{\psi}_{3}(\mathrm{t})=\operatorname{Sech}(\mathrm{t})$.

Figure $4 \mathrm{~b}$ represents the energy $\varepsilon_{k}$ in this case, everything else being as in Fig. 4a. The waves with energy $\varepsilon_{1}$ and $\varepsilon_{3}$ decay continuously to zero. Their energy is gained by the third, which ends up with all the energy of the system.

\subsection{Phase velocity}

The basic wave phase velocity derived from the dispersion Eq. (7) is expressed as:

$C=U-\frac{\beta-\left(d^{2} U / d y^{2}\right)}{\rho^{2}}$.

The dependence of this phase velocity on the mean zonal wind $\mathrm{U}(\mathrm{y})$ indicates that tropical waves propagate essentially 


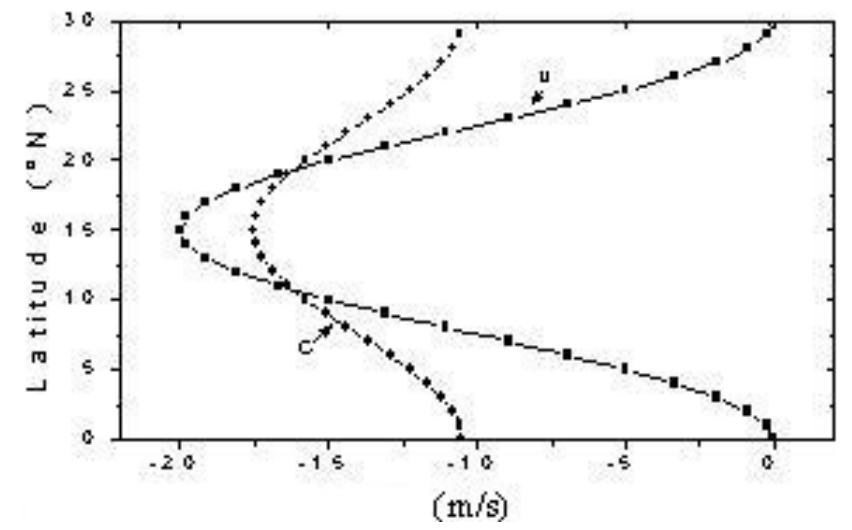

Fig. 5. Meridional dependence of the phase velocity, $C$, and mean zonal wind, U. Negative values indicate that the waves propagate westward.

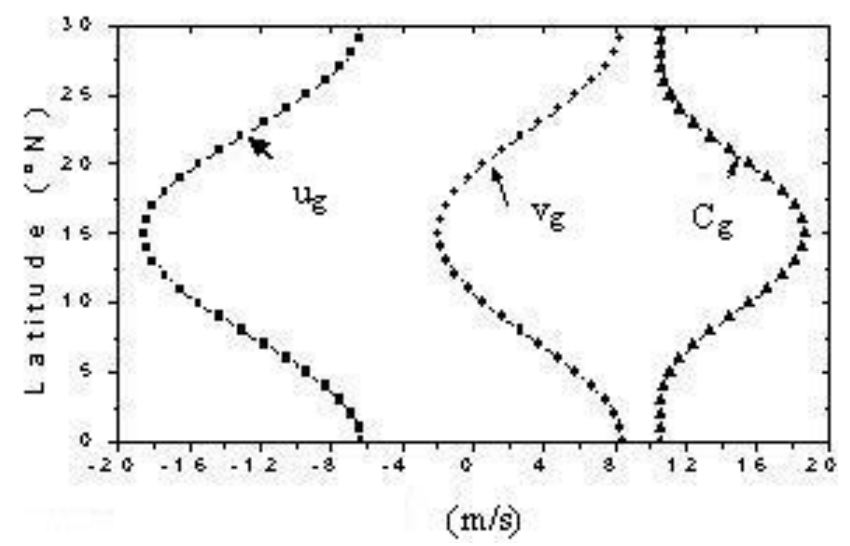

Fig. 6. Meridional dependence of the group velocity $\left(\mathrm{C}_{g}\right)$ and its zonal $\left(\mathrm{u}_{g}\right)$ and meridional $\left(\mathrm{v}_{g}\right)$ components in the presence of tropical instability.

westward at this level. The latitude dependence of the velocity illustrates the nonlinear effects. Furthermore, the velocity $\mathrm{C}$ is also a function of zonal and meridional wave numbers, decreasing as the latter increases. This result is in agreement with Holton (1979), who showed that the advection of planetary vorticity, which tends to dampen fluctuations, dominates the advection of relative vorticity as the wavelength of the perturbation increases. Figure 5 illustrates the profile of $\mathrm{C}(\mathrm{y})$ for a tropical instability, with zonal and meridional wavelength values of $6000 \mathrm{~km}$ and $3000 \mathrm{~km}$, respectively, and $\beta$ (the meridional gradient of the Coriolis parameter) taken as $2.2 \times 10^{-11} \mathrm{~m}^{-2} \mathrm{~s}^{-1}$ at $15^{\circ} \mathrm{N}$. The profile is symmetric with respect to the jet axis, with the instability maximum on it. Thus, Rossby waves are slower than the mean circulation. The meridional mean value of the phase velocity is of the order of $-14 \mathrm{~m} \cdot \mathrm{s}^{-1}$ and the resultant wavelength is set equal to $\lambda=\frac{2 \pi}{\rho}(2700 \mathrm{~km})$. These results compare quite well with those obtained around the AEJ.

\subsection{Group velocity}

The propagation of AEWs results from the superposition of three fundamental waves whose wave numbers, growth rates and amplitude are tied together by Eqs. (6), (7) and (14). Hence, we have a wave packet moving with a group velocity corresponding to the propagation of system energy in the horizontal plane.

The zonal component, $\mathrm{u}_{g}$, and meridional component, $\mathrm{v}_{g}$, of group velocity, $\boldsymbol{C}_{g}$, are obtained from Eq. (7) through the following expressions:

$u_{g}=U-\frac{\beta-\left(d^{2} U / d y^{2}\right)}{\rho^{2}}+2\left(\beta-\frac{d^{2} U}{d y^{2}}\right) \frac{m^{2}}{\rho^{4}}$.

and

$v_{g}=2\left(\beta-\frac{d^{2} U}{d y^{2}}\right) \frac{m \cdot n}{\rho^{4}}$

Following Hoskin et al. (1993), one can define a unitary vector, $\rho$, normal to the wavefront, which has a positive component eastward and makes an angle $\theta$ relative to this direction and has zonal and meridional vectors $\boldsymbol{m}$ and $\boldsymbol{n} . \boldsymbol{C}_{g}$ is then rewritten as:

$\vec{C}_{g}=u_{g} \frac{\vec{m}}{m}+v_{g} \frac{\vec{n}}{n}$

or

$\vec{C}_{g}=(C, O)+2\left(\beta-\frac{d^{2} U}{d y^{2}}\right) \frac{\cos \theta}{\rho^{2}} \vec{\rho}$,

where $\mathrm{C}$ is the perturbation phase velocity as described by Eq. (15).

In the case of stationary waves, we have $\sigma=0$, and $\mathrm{C}=0$, since $\mathrm{C}=\sigma / \mathrm{m}$. Using Eq. (15), one can write:

$\rho^{2}=\rho_{s}^{2}=\frac{\beta-\left(d^{2} U / d y^{2}\right)}{U}$.

Thus, the group velocity vector takes the following form in the case of stationary waves:

$\vec{C}_{g}=C_{g} \vec{\rho}$

with

$C_{g}=2\left(\beta-\frac{d^{2} U}{d y^{2}}\right) \frac{\cos \theta}{\rho_{s}^{2}}=2 U \cos \theta$.

The frontal zone of stationary waves moves with a group velocity equal to $2 \mathrm{U} \cos \theta$. This velocity is double the zonal wind $U$ when the perturbation is moving in the zonal direction, and is zero when the perturbation is moving northward.

But, as Fig. 5 clearly shows, the value of the phase velocity around the lower African tropospheric jet is in the interval -10.5 to $-17.5 \mathrm{~m} . \mathrm{s}^{-1}$, indicating that there are no stationary waves in this case. However, the technique presented here is applicable to the special case of westerly waves or to the attenuated easterly waves (Hoskin and Ambrizzi, 1993). 
Figure 6 shows the values of the group velocity $(\mathrm{Cg})$ and their zonal and meridional components as functions of latitude. The negative value indicates that the energy of the perturbation is moving south-westward. The magnitude of the group velocity vector is, as $\mathrm{U}(\mathrm{y})$ and $\mathrm{C}(\mathrm{y})$, bell shaped and centred around the jet axis. The energy essentially moves westward in the zonal direction, where $\mathrm{C}_{g}$ takes its maximum value $\left(-18 \mathrm{~m} \cdot \mathrm{s}^{-1}\right)$ around the jet core, and southward in the region between 11 to $19^{\circ} \mathrm{N}$.

\section{Conclusion}

We have studied the structure of AEW in the lower troposphere of the tropical zone, for the specific case of weak interactions at resonance. The weak interaction approximation appears to be an interesting approach to the study of tropical instabilities. The results of the approximation, which is "midway" between nonlinear and linear treatments, are in agreement with Dobryshman (1982) and Vanneste and Vial (1995), especially concerning the phase velocity and its meridional variations. It is also shown that the energy of the waves moves essentially westward with a group velocity of around $18.6 \mathrm{~m} . \mathrm{s}^{-1}$ in the jet core, where the basic state moves essentially westward, at $20 \mathrm{~m} \cdot \mathrm{s}^{-1}$. Hence, for a strong easterly mean zonal wind, we see that the AEW cannot be stationary. However, one can obtain nonlinear stationary waves by defining a new frame of reference in which the waves are fixed. These simplifications lead, in some cases, to solitary waves (Rottman et al., 1993). The effects of the nonlinearity, controlled by the wave amplitudes $\psi_{k}(\mathrm{t})$, provides a possible mechanism for energy transfer between waves, as well as allow for the calculation of the interaction period which is equal to those of AEW.

How can these nonlinear theories of wave trapping be understood, which carry over to the case of nonlinear solitary waves? And what is the impact on the AEW? Atmospheric solitary waves are horizontally propagating nonlinear waves that can travel over large distances with minimal change in form as the result of balance between nonlinearity and linear dispersion. Then, an estimate for the wave amplitude is usually used for comparing the weakly nonlinear theory for steadily propagating solitary waves with atmospheric observations (Christine, 1992; Rottman and Einaudi, 1993).

We have reviewed attempts to use the simplest forms of weak interaction to predict the character to the AEW. In particular, the theory requires an understanding of what mechanism is trapping the waves. Since this is difficult to determine in practice, the observed waves are often strongly nonlinear (Thorncroft and Hoskins, 1995) and there is clearly a need to develop theories which can take this into account.

\section{References}

Abromowitz, M. and Segun Irene A.: Handbook of Mathematical Function, Dover Pub., Inc., New York, 1964.

Burpee, R. W.: The origin and structure of easterly waves in the lower troposphere of North Africa. J. Atmos. Sci., 29, 77-90, 1972.

Christine, D. R.: The morning glory of the Gulf of Carpentaria: A paradigm for nonlinear waves in the lower troposphere. Aust. Meteor. Mag., 41, 21-60, 1992.

Dobryshman, E. M.: Theoretical studies of tropical waves. GATE Pub., 25, 121-177, 1982.

Ferreira, R. N. and Schubert, W. H.: Barotropic aspects of ITCZ breakdown. J. Atmos. Sci, 52(2), 261-285, 1997.

Holton, J. R.: An introduction to dynamic meteorology. Inter. Geoph. Series16, 2nd ed., 1979.

Hoskins, B. J. and Ambrizzi, T.: Rossby wave propagation on a realistic longitudinally varying flow. J. Atmos. Sci, 50, 1661$1671,1993$.

Kadomtsev, B. B.: Phénomènes collectifs dans le plasmas. Ed. MIR, Moscou, 161-187, 1979.

Krishnamurti, T. N. and Pasch, R. J.: Large-scale mean state. GATE Pub., 25, 27-54, 1982.

Kwon, H. J.: A re-examination of Genesis of African wave. J. Atmos. Sci, 46, 3621-3631, 1989.

Longuet-Higgins, M. C. and Hill A. E.: Resonance interaction of the planetary waves in nonlinear theory of wave propagation Ed. MIR, Moscou, 161-187, 1970.

Mass, C.: A linear equation model of African wave disturbances. J. Atmos. Sci, 36, 2075-2092, 1979.

Mc Intyre, M. E. and Palmer T. N.: Breaking planetary waves in the stratosphere. Nature, 305, 593-599, 1983.

Norquist, D. E., Recker, E. E., and Reed, R. J.: The energetic of African wave disturbances as observed during phase III of GATE. Mon. Wea. Rev., 105, 334-342, 1977.

Pedlosky, J.: Geophysical Fluid Dynamics, Springer Verlag; 2nd ed., 1998.

Rennick, A. M.: The generation of African wave. J. Atmos. Sci, 33, 1955-1969. 1976.

Rottman, J. W. and Einaudi, F.: Solitary waves in the atmosphere J. Atmos. Sci., 50, 2116-2131, 1993.

Thorncroft, C. D. and Hoskins, B. J.: An idealised study of African easterly waves. Part 1: A linear view. Q. J. R. Meteorol. Soc., 120, 953-982, 1994a.

Thorncroft, C. D. and Hoskins, B. J.: An idealised study of African easterly waves. Part 2: A nonlinear view. Q. J. R. Meteorol. Soc., 120, 983-1015, 1994b.

Thorncroft, C. D. and Hoskins, B. J.: An idealised study of African easterly waves. Part 3: More realistic basic states. Q. J. R. Meteorol. Soc., 121, 1589-1614, 1995.

Vanneste, J. and Vial, F.: On the nonlinear interaction of geophysical waves in shear flows, Geophys. and Astrophys. Fluid Dynamics, 78, 115-141, 1995.

Wegiel, J. W. and Herbster, C. C.: The origin of African easterly waves? Department of Meteorology. Tallahassee, FL 323044520, 2000.

Acknowledgements. This work was partially funded by ACMAD/FIRMA Grant FIRMA-II-B 12/7.

Topical Editor O. Boucher thanks two referees for their help in evaluating this paper. 